\title{
A Systematic Review and Meta-Analysis of Epidemiology and Clinical Manifestations of Human Brucellosis in China
}

\author{
Rongjiong Zheng, ${ }^{1}$ Songsong Xie, ${ }^{1}$ Xiaobo Lu, ${ }^{1}$ Lihua Sun ${ }^{D},{ }^{1}$ Yan Zhou, \\ Yuexin Zhang $\mathbb{D},{ }^{1}$ and Kai Wang $\mathbb{D}^{2}$ \\ ${ }^{1}$ Department of Infectious Diseases, The First Affiliated Hospital of Xinjiang Medical University, Urumqi, Xinjiang 830054, China \\ ${ }^{2}$ Department of Medical Engineering and Technology, Xinjiang Medical University, Urumqi, Xinjiang 830011, China
}

Correspondence should be addressed to Yuexin Zhang; zhangyx3103@163.com and Kai Wang; wangkaimath@sina.com

Received 30 November 2017; Accepted 1 March 2018; Published 22 April 2018

Academic Editor: Vincenzo Veneziano

\begin{abstract}
Copyright (C) 2018 Rongjiong Zheng et al. This is an open access article distributed under the Creative Commons Attribution License, which permits unrestricted use, distribution, and reproduction in any medium, provided the original work is properly cited.

Background. Brucellosis has a wide spectrum of clinical manifestations and it may last several days or even several years; however, it is often misdiagnosed and therefore may cause inadequate therapy and prolonged illness. Previous studies about meta-analysis of manifestations of brucellosis reported in English lacked the data published in Chinese, which did not provide details about the contact history, laboratory tests, and misdiagnosis. We undertake a meta-analysis of clinical manifestations of human brucellosis in China to identify those gaps in the literature. We have searched published articles in electronic databases up to December 2016 identified as relating to clinical features of human brucellosis in China. 68 studies were included in the analysis. The main clinical manifestations were fever, fatigue, arthralgia, and muscle pain $(87 \%, 63 \%, 62 \%$, and $56 \%$, resp.). There are significant differences between adults and children. Rash, respiratory and cardiac complications, and orchitis/epididymitis were more prevalent in children patients. The common complications of brucellosis were hepatitis, followed by osteoarthritis, respiratory diseases, cardiovascular diseases, central nervous system dysfunction, hemophagocytic syndrome, and orchitis/epididymitis in male. In the nonpastoral areas, brucellosis has a high ratio of misdiagnosis. Our analysis provides further evidence for the accurate diagnosis, particularly in assessing severe, debilitating sequelae of this infection.
\end{abstract}

\section{Introduction}

Brucellosis is one of the most common zoonotic infections globally $[1,2]$. The disease is transmitted to humans by direct/indirect contact with infected animals or through the consumption of raw meat and dairy products $[3,4]$. The main transmission routes are digestive tract, skin, and mucosal and respiratory tract contact with blood body fluids and aerosols.

Brucellosis has a wide spectrum of clinical manifestations, often lacks specificity, may last from several days to more than a year, is often misdiagnosed, and therefore causes inadequate therapy and prolonged illness can cause a severely debilitating and disabling illness. Patients may show fever, sweating, fatigue, and osteoarthritis [5] and even more serious conditions in different organ systems [6]. Brucellosis not only causes huge economic loss to the society by influencing the production of animal husbandry, but also threatens the human's physical and mental health [7].

Brucellosis was first reported in China in 1905 [8]. In recent years, human brucellosis incidence has increased sharply $[9,10]$. Nationwide surveillance data indicated that the total incidence rate of human brucellosis in mainland China increased from 0.92 cases $/ 100,000$ people in 2004 to 4.2 cases/100,000 people in 2014 [11-13]. Currently, human brucellosis remains one of major public health issues in China.

This study presents a systematic review of scientific literature published before December 2016 identified as relating to clinical features of brucellosis in China. The objectives of this review were to identify those gaps in the literature of epidemiology, clinical manifestations, contact history, laboratory tests, and misdiagnosis of human brucellosis in 
China and provide further evidence for the accurate diagnosis, particularly in assessing severe, debilitating sequelae of human brucellosis.

\section{Methods}

2.1. Search Strategy. We performed a systematic review of the literature to identify articles relating to clinical features of human brucellosis in China. With assistance of a professional medical librarian we electronically searched the literature in Wan Fang Data, Wei Pu Data, CNKI, Medline, Cochrane Library, and PubMed with MESH and keyword subject headings "brucellosis," "malta fever," "brucella melitensis," or "brucella abortus," AND "symptom," "sequelae," "morbidity," "mortality," "transmission mode," "foodborne," and "China," for entries published from databases' inception before December 2016. We did not restrict the types of studies and publication languages. Duplicate entries were identified by two investigators screening the titles and abstracts of the article, the author, the year of publication, and the volume, issue, and page numbers of the source, and reviewing potentially relevant articles in full.

2.2. Selection Criteria. We systematically and inclusively reviewed articles by two investigators. The reviewers selected articles first by title and abstract, next by full text, and last by analyzing eligible studies in detail until demonstrating $100 \%$ agreement in articles included and excluded by two investigators.

Studies with the following criteria were excluded: (A) articles related to non-human brucellosis; (B) reported data that overlapped with already included articles; (C) articles that could not provide original data of the patients; (D) articles addressing topics that were not related to the clinical features of human brucellosis, such as treatment intervention and experimental laboratory studies.

Studies with the following criteria were included: (A) the literatures that described the clinical symptoms/syndromes of human brucellosis and the number of study subjects must more than 10 in each document; (B) the subjects reported in the literature who must be in China; $(\mathrm{C})$ studies that provided data from general brucellosis cases and presented relevant laboratory results.

2.3. Data Extraction. Data was extracted by two reviewers independently including data collection, study design, study location, patient characteristics, the number of male and female patients, clinical manifestations, numbers of subjects with each symptom and complication which were recorded for each study, methods of diagnosis, and laboratory parameters. For the sex-related outcomes of epididymo/orchitis, the study population was considered to be only the male subgroups of the study population. Children patients must be of the age of $0-15$ years. We also recorded the information relating to duration of illness prior to treatment, diagnostic delay, and exposure to potential risk factors. The results of data extraction must reach an agreement and consensus between the reviewers.

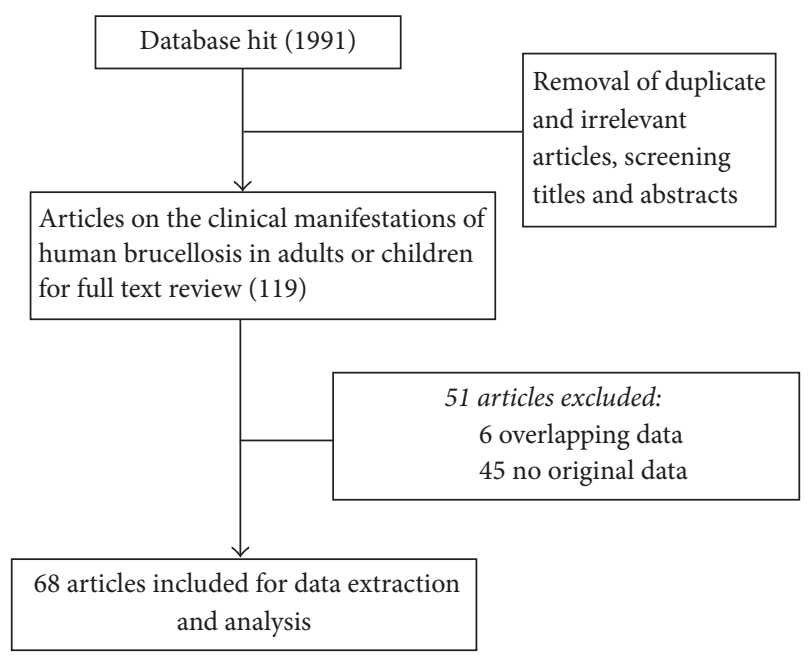

FIGURE 1: Procedure of the selection process.

2.4. Statistical Analyses. We defined an event rate as the ratio of number of reported cases with a specific clinical manifestation to the total number of reported cases in each study. $\mathrm{R}$ statistical software (version 3.4.2, meta package) will be used for creating Forest plots to summarize composite data, generating proportions and corresponding 95\% confidence intervals for each manifestation. Two-sided $P$ values $<0.05$ will be considered statistically significant during hypothesis testing.

\section{Results}

3.1. Systematic Review. Literature searches yielded 1991 potential articles, leaving 68 publications that met inclusion and exclusion criteria for data extraction and final analyses. 68 studies represented 12842 patients with human brucellosis in China. The male: female ratio was 2.64:1. All 68 articles included in the analysis were case series studies. Figure 1 illustrates the detailed search process.

Studies selected from 20 provinces or autonomous regions of China, including 39 studies from pastoral areas (12 from Xinjiang, 9 from Heilongjiang, 7 from Inner Mongolia, 4 from Jilin, 4 from Ningxia, 2 from Gansu, and 1 from Liaoning) and 29 studies from nonpastoral areas (6 from Shandong, 5 from Beijing, 3 from Henan, 3 from Shanxi, 2 from Hebei, 2 from Shaanxi, 2 from Tianjin, 1 from Guangdong, 1 from Hunan, 1 from Jiangsu, 1 from Jiangxi, 1 from Yunnan, and 1 from Zhejiang). The geographic distributions of the numbers of subjects from each selected study are shown in Figure 2.

We identified 41 studies which included both children and adult patients [14-54].10 studies investigated children with an upper age limit ranging from 0 months to 15 years [55-64]. 17 studies were about the adults who are more than 15 years old [65-81]. The results are presented in detail in Table 1.

3.2. Contact History. 54 studies provided data about contact history (see Table 2). Most of the patients (79.4\% [95\% CI $76.5 \%-82.4 \%]$ ) had histories of closely contacting with 
TABLE 1: Main characteristics of all studies included in the meta-analysis.

\begin{tabular}{|c|c|c|c|c|c|c|c|c|}
\hline $\begin{array}{l}\text { First author \& } \\
\text { ref. number }\end{array}$ & Year & $\begin{array}{l}\text { Age } \\
\text { category }\end{array}$ & Location & Cases & $\begin{array}{c}\text { Available } \\
\text { contact history } \\
\text { data }\end{array}$ & $\begin{array}{c}\text { Available } \\
\text { laboratory data }\end{array}$ & $\begin{array}{c}\text { Available blood } \\
\text { culture data }\end{array}$ & $\begin{array}{c}\text { Available } \\
\text { misdiagnosis } \\
\text { data }\end{array}$ \\
\hline Wu et al. [14] & 2012 & All ages & Beijing & 44 & Yes & NA & NA & Yes \\
\hline Dai et al. [15] & 2013 & All ages & Beijing & 23 & Yes & Yes & Yes & NA \\
\hline Ge et al. [16] & 2011 & All ages & Beijing & 66 & Yes & Yes & Yes & Yes \\
\hline Tong et al. [17] & 2013 & All ages & Beijing & 35 & Yes & NA & NA & NA \\
\hline Guo and $\mathrm{Xu}[18]$ & 2013 & All ages & Beijing & 21 & Yes & Yes & Yes & Yes \\
\hline Wang et al. [19] & 2015 & All ages & Gansu & 61 & Yes & Yes & NA & NA \\
\hline Gao et al. [20] & 2002 & All ages & Gansu & 182 & Yes & NA & NA & NA \\
\hline Zhang et al. [21] & 2012 & All ages & Henan & 21 & Yes & Yes & Yes & NA \\
\hline Li et al. [22] & 2016 & All ages & Henan & 905 & Yes & NA & NA & NA \\
\hline Zhou [23] & 2009 & All ages & Henan & 241 & NA & NA & NA & Yes \\
\hline Li et al. [24] & 2008 & All ages & Heilongjiang & 165 & Yes & Yes & Yes & NA \\
\hline $\begin{array}{l}\text { Liu and Zhang } \\
\text { [25] }\end{array}$ & 2016 & All ages & Inner Mongolia & 44 & NA & NA & NA & NA \\
\hline Liu et al. [26] & 2015 & All ages & Heilongjiang & 314 & NA & Yes & Yes & NA \\
\hline Meng et al. [27] & 2015 & All ages & Heilongjiang & 3318 & NA & NA & Yes & NA \\
\hline Gong et al. [28] & 2010 & All ages & Heilongjiang & 1470 & NA & NA & NA & NA \\
\hline Liu et al. [29] & 2012 & All ages & Heilongjiang & 229 & Yes & Yes & Yes & Yes \\
\hline Sun et al. [30] & 2010 & All ages & Jilin & 270 & Yes & NA & NA & NA \\
\hline Wang et al. [31] & 2014 & All ages & Liaoning & 88 & Yes & NA & NA & NA \\
\hline Xie et al. [32] & 2016 & All ages & Inner Mongolia & 166 & NA & NA & NA & NA \\
\hline $\begin{array}{l}\text { Sheng and Ma } \\
\text { [33] }\end{array}$ & 2009 & All ages & Inner Mongolia & 829 & NA & NA & NA & NA \\
\hline Sun et al. [34] & 2014 & All ages & Inner Mongolia & 126 & Yes & Yes & Yes & NA \\
\hline $\begin{array}{l}\text { W. Yang and F. } \\
\text { Yang [35] }\end{array}$ & 2015 & All ages & Inner Mongolia & 228 & Yes & Yes & NA & Yes \\
\hline Duan [36] & 2015 & All ages & Ningxia & 57 & Yes & NA & NA & NA \\
\hline $\begin{array}{l}\text { Zhang and } \\
\text { Wang [37] }\end{array}$ & 2013 & All ages & Ningxia & 128 & Yes & NA & NA & Yes \\
\hline Wang [38] & 2005 & All ages & Shandong & 62 & Yes & NA & NA & NA \\
\hline $\begin{array}{l}\text { Wang and } \\
\text { Xiong [39] }\end{array}$ & 2011 & All ages & Shandong & 235 & NA & Yes & NA & Yes \\
\hline Lian et al. [40] & 2015 & All ages & Shandong & 232 & Yes & NA & NA & Yes \\
\hline Gao [41] & 2016 & All ages & Shandong & 94 & Yes & NA & NA & NA \\
\hline Wang et al. [42] & 2014 & All ages & Shandong & 96 & Yes & NA & Yes & Yes \\
\hline Wang [43] & 2010 & All ages & Shanxi & 86 & Yes & NA & NA & NA \\
\hline $\begin{array}{l}\text { Feng and Deng } \\
{[44]}\end{array}$ & 2016 & All ages & Shanxi & 105 & Yes & Yes & Yes & Yes \\
\hline An et al. [45] & 2001 & All ages & Shaanxi & 622 & Yes & NA & NA & NA \\
\hline Zhang et al. [46] & 2016 & All ages & Yunnan & 43 & Yes & Yes & Yes & NA \\
\hline Guo et al. [47] & 2016 & All ages & Xinjiang & 124 & Yes & NA & NA & NA \\
\hline Pan et al. [48] & 2013 & All ages & Xinjiang & 153 & Yes & Yes & NA & NA \\
\hline $\begin{array}{l}\text { Zhang and Liu } \\
\text { [49] }\end{array}$ & 2013 & All ages & Xinjiang & 57 & Yes & Yes & Yes & Yes \\
\hline Yang et al. [50] & 2015 & All ages & Xinjiang & 125 & NA & NA & NA & Yes \\
\hline Zhang [51] & 2016 & All ages & Xinjiang & 191 & Yes & Yes & NA & NA \\
\hline Ju et al. [52] & 2011 & All ages & Xinjiang & 156 & Yes & Yes & Yes & NA \\
\hline Gao et al. [53] & 2012 & All ages & Xinjiang & 426 & NA & NA & NA & NA \\
\hline Wang et al. [54] & 2015 & All ages & Xinjiang & 117 & Yes & Yes & Yes & NA \\
\hline Wang et al. [55] & 2014 & Children & Hebei & 80 & Yes & NA & NA & NA \\
\hline
\end{tabular}


TABLE 1: Continued.

\begin{tabular}{|c|c|c|c|c|c|c|c|c|}
\hline $\begin{array}{l}\text { First author \& } \\
\text { ref. number }\end{array}$ & Year & $\begin{array}{c}\text { Age } \\
\text { category }\end{array}$ & Location & Cases & $\begin{array}{c}\text { Available } \\
\text { contact history } \\
\text { data }\end{array}$ & $\begin{array}{c}\text { Available } \\
\text { laboratory data }\end{array}$ & $\begin{array}{l}\text { Available blood } \\
\text { culture data }\end{array}$ & $\begin{array}{c}\text { Available } \\
\text { misdiagnosis } \\
\text { data }\end{array}$ \\
\hline Zeng et al. [56] & 2014 & Children & Jilin & 23 & Yes & Yes & Yes & Yes \\
\hline Wang et al. [57] & 2016 & Children & Xinjiang & 16 & Yes & Yes & NA & NA \\
\hline Fan et al. [58] & 2016 & Children & Xinjiang & 24 & Yes & Yes & $\mathrm{NA}$ & NA \\
\hline Zhang et al. [59] & 2006 & Children & Jilin & 25 & $\mathrm{NA}$ & Yes & $\mathrm{NA}$ & $\mathrm{NA}$ \\
\hline Yu et al. [60] & 2012 & Children & Heilongjiang & 38 & NA & Yes & NA & NA \\
\hline Lu and Liu [61] & 2015 & Children & Inner Mongolia & 17 & Yes & Yes & NA & NA \\
\hline Liu et al. [62] & 2016 & Children & Heilongjiang & 94 & Yes & Yes & Yes & Yes \\
\hline $\begin{array}{l}\text { Bai and Duan } \\
{[63]}\end{array}$ & 2015 & Children & Ningxia & 48 & Yes & Yes & Yes & Yes \\
\hline $\mathrm{He}[64]$ & 2015 & Children & Xinjiang & 19 & Yes & Yes & Yes & Yes \\
\hline Zheng et al. [65] & 2016 & Adults & Guangdong & 12 & Yes & Yes & Yes & $\mathrm{NA}$ \\
\hline Tong et al. [66] & 2008 & Adults & Hebei & 25 & Yes & Yes & Yes & Yes \\
\hline $\begin{array}{l}\text { Chen and Dong } \\
{[67]}\end{array}$ & 2016 & Adults & Heilongjiang & 60 & Yes & NA & NA & NA \\
\hline Huang [68] & 2016 & Adults & Hunan & 17 & Yes & Yes & Yes & Yes \\
\hline Ji et al. [69] & 2006 & Adults & Heilongjiang & 30 & NA & Yes & Yes & NA \\
\hline $\begin{array}{l}\text { M. Wang and L. } \\
\text { Wang [70] }\end{array}$ & 2007 & Adults & Jilin & 26 & Yes & Yes & NA & Yes \\
\hline Zhang et al. [71] & 2016 & Adults & Jiangsu & 39 & Yes & Yes & Yes & Yes \\
\hline Guo et al. [72] & 2016 & Adults & Jiangxi & 12 & Yes & Yes & Yes & Yes \\
\hline Zhang [73] & 2011 & Adults & Inner Mongolia & 27 & Yes & Yes & NA & $\mathrm{NA}$ \\
\hline Yan et al. [74] & 2016 & Adults & Ningxia & 31 & NA & Yes & Yes & NA \\
\hline Li et al. [75] & 2015 & Adults & Shandong & 21 & Yes & Yes & Yes & Yes \\
\hline Wu et al. [76] & 2007 & Adults & Shanxi & 28 & Yes & Yes & NA & NA \\
\hline $\begin{array}{l}\text { Zhang and } \mathrm{Li} \\
{[77]}\end{array}$ & 2015 & Adults & Shaanxi & 35 & Yes & Yes & Yes & NA \\
\hline Wang [78] & 2014 & Adults & Tianjin & 17 & Yes & Yes & Yes & Yes \\
\hline $\begin{array}{l}\text { Zhou and Yang } \\
\text { [79] }\end{array}$ & 2014 & Adults & Tianjin & 18 & Yes & Yes & Yes & $\mathrm{NA}$ \\
\hline Xu et al. [80] & 2007 & Adults & Zhejiang & 31 & Yes & NA & NA & $\mathrm{NA}$ \\
\hline Chen et al. [81] & 2016 & Adults & Xinjiang & 74 & Yes & Yes & NA & NA \\
\hline
\end{tabular}

TABLE 2: Meta-analysis of the contact history.

\begin{tabular}{lcr}
\hline Contact & $n$ & Proportion [95\% CI] \\
\hline Contact history & 54 & $0.794[0.7651 ; 0.8240]$ \\
Digestive tract contact & 31 & $0.115[0.0844 ; 0.1567]$ \\
Unknown & 43 & $0.167[0.1347 ; 0.2077]$ \\
\hline
\end{tabular}

cattle, sheep, pigs, and dogs. $11.5 \%$ (95\% CI 8.4\%-15.7\%) cases had consumption history of uncooked meat or dairy products. $16.7 \%$ (95\% CI 13.5\%-20.8\%) cases got the infection of brucellosis with unknown reason. The brucellosis is mostly associated with direct/indirect contact with infected animals or through the consumption of animal products in China.

3.3. Clinical Syndromes and Complications. Table 3 shows the clinical syndromes and complications of patients by age category. There are 17 articles specifically describing the clinical characteristics of adult brucellosis representing 503 patients (male 408, female 95). Fever was the most common clinical syndrome (pooled rate 99\% [95\% CI 97\%-100\%]), followed by muscle pain (76\% [95\% CI 60\%-95\%]), fatigue (64\% [95\% CI 55\%-74\%]), arthralgia (61\% [95\% CI 52\%-70\%]), and sweating (57\% [95\% CI 48\%-68\%]). 10 articles specifically describe the clinical characteristics of children brucellosis including 384 patients (male 249, female 135). The most common symptoms of children patients were fever $(92 \%$ [95\% CI 87\%-97\%]), fatigue (68\% [95\% CI 56\%-83\%]), sweating $(60 \%$ [95\% CI 45\%-79\%]), and arthralgia $(52 \%$ [95\% CI 43\%-64\%]). The remaining 41 articles include 
TABLE 3: Meta-analysis of clinical manifestations of brucellosis by age category.

\begin{tabular}{|c|c|c|c|c|c|c|c|c|}
\hline \multirow{3}{*}{$\begin{array}{l}\text { Manifestation } \\
\text { General }\end{array}$} & \multicolumn{6}{|c|}{ Age category } & \multirow{2}{*}{\multicolumn{2}{|c|}{ All studies }} \\
\hline & \multicolumn{2}{|c|}{ Children } & \multicolumn{2}{|c|}{ Adults } & \multicolumn{2}{|c|}{ All ages } & & \\
\hline & $n$ & $\%[95 \% \mathrm{CI}]$ & $n$ & $\%$ [95\% CI] & $n$ & $\%[95 \% \mathrm{CI}]$ & $n$ & $\%[95 \% \mathrm{CI}]$ \\
\hline Fever & 10 & $92[87 ; 97]$ & 17 & $99[97 ; 100]$ & 41 & $83[80 ; 87]$ & 68 & $87[85 ; 90]$ \\
\hline Fatigue & 7 & $68[56 ; 83]$ & 14 & $64[55 ; 74]$ & 34 & $62[57 ; 67]$ & 55 & $63[59 ; 67]$ \\
\hline Chills & 3 & $26[8 ; 82]$ & 5 & $53[36 ; 79]$ & 4 & $37[33 ; 42]$ & 12 & $43[33 ; 55]$ \\
\hline Sweats & 8 & $60[45 ; 79]$ & 16 & $57[48 ; 68]$ & 39 & $54[49 ; 59]$ & 63 & $55[51 ; 60]$ \\
\hline Arthralgia & 9 & $52[43 ; 64]$ & 17 & $61[52 ; 70]$ & 40 & $63[59 ; 68]$ & 66 & $62[58 ; 65]$ \\
\hline Headache & 4 & $8[3 ; 19]$ & 10 & $29[19 ; 42]$ & 27 & $21[18 ; 25]$ & 41 & $21[18 ; 25]$ \\
\hline Muscle pain & 2 & $31[7 ; 100]$ & 5 & $76[60 ; 95]$ & 20 & $53[47 ; 59]$ & 27 & $56[51 ; 62]$ \\
\hline Nausea/vomiting & 6 & $27[16 ; 43]$ & 8 & $26[15 ; 45]$ & 17 & $25[19 ; 34]$ & 31 & $26[21 ; 33]$ \\
\hline Rash & 3 & $13[6 ; 29]$ & 3 & $7[3 ; 19]$ & 9 & $5[3 ; 11]$ & 15 & $7[4 ; 11]$ \\
\hline Weight loss & 0 & - & 4 & $26[14 ; 47]$ & 5 & $32[17 ; 61]$ & 9 & $29[17 ; 48]$ \\
\hline Skin petechia & 3 & $8[4 ; 18]$ & 2 & $18[10 ; 32]$ & 9 & $5[3 ; 8]$ & 14 & $7[4 ; 10]$ \\
\hline Abdominal pain & 2 & $6[1 ; 31]$ & 3 & $6[3 ; 14]$ & 3 & $8[4 ; 16]$ & 8 & $8[5 ; 11]$ \\
\hline Chest pain & 0 & - & 2 & $7[3 ; 17]$ & 1 & $5[3 ; 10]$ & 3 & $6[3 ; 10]$ \\
\hline Cough & 5 & $12[8 ; 17]$ & 4 & $19[12 ; 29]$ & 5 & $10[8 ; 14]$ & 14 & $12[10 ; 15]$ \\
\hline Hepatomegaly & 7 & $28[18 ; 42]$ & 7 & $23[13 ; 40]$ & 23 & $13[10 ; 17]$ & 37 & $16[13 ; 20]$ \\
\hline Splenomegaly & 7 & $35[27 ; 45]$ & 10 & $29[22 ; 39]$ & 23 & $21[16 ; 27]$ & 40 & $24[20 ; 29]$ \\
\hline Lymphadenectasis & 7 & $38[25 ; 58]$ & 7 & $32[22 ; 48]$ & 27 & $16[12 ; 21]$ & 41 & $19[15 ; 25]$ \\
\hline Hepatitis & 8 & $48[34 ; 67]$ & 15 & $60[52 ; 69]$ & 24 & $38[30 ; 49]$ & 47 & $45[38 ; 54]$ \\
\hline Neurological & 4 & $8[4 ; 17]$ & 3 & $8[2 ; 36]$ & 14 & $4[2 ; 9]$ & 21 & $5[3 ; 10]$ \\
\hline Cardiac & 3 & $19[2 ; 100]$ & 2 & $5[1 ; 19]$ & 12 & $9[6 ; 14]$ & 17 & $9[6 ; 16]$ \\
\hline Hemophagocytic syndrome & 0 & - & 0 & - & 4 & $6[2 ; 23]$ & 4 & $6[2 ; 23]$ \\
\hline Respiratory & 5 & $26[12 ; 57]$ & 3 & $11[6 ; 20]$ & 8 & $9[4 ; 23]$ & 16 & $13[7 ; 21]$ \\
\hline Orchitis/epididymitis & 1 & $67[45 ; 100]$ & 7 & $6[3 ; 12]$ & 34 & $9[7 ; 12]$ & 42 & $9[7 ; 12]$ \\
\hline Osteoarthritis & 2 & $16[8 ; 35]$ & 4 & $22[9 ; 52]$ & 11 & $23[17 ; 31]$ & 17 & $22[17 ; 29]$ \\
\hline
\end{tabular}

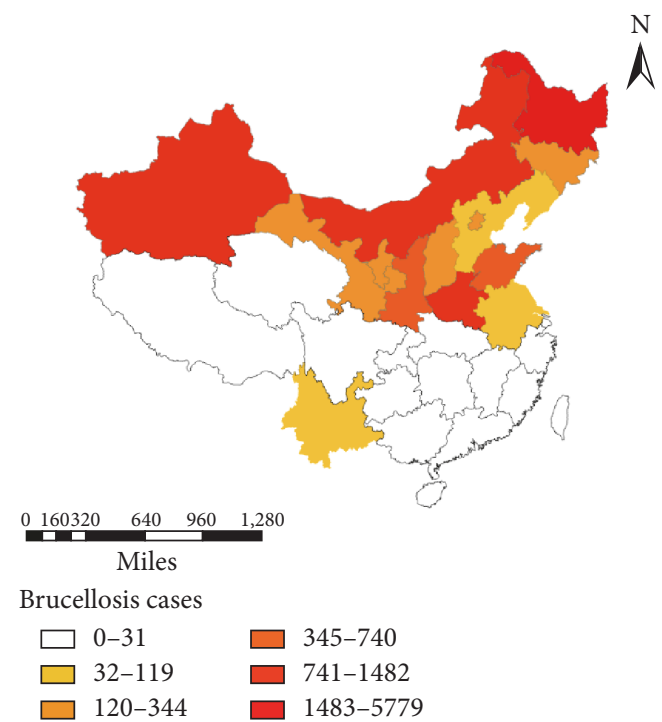

FIGURE 2: The geographic distribution of the numbers of subjects from each selected study.

pediatric and adult patients, a total of 11955 cases (male 8654, female 3301). Children patients have a higher incidence rate of rash, respiratory and cardiac complications, and orchitis/epididymitis. The morbidity of chills, headache, and weight loss are lower compared to adults.

Hepatitis (45\% [95\% CI 38\%-54\%]) and osteoarthritis $(22 \%$ [95\% CI $17 \%-29 \%])$ were the most common complications. Central nervous system dysfunction (5\% [95\% CI 3\%-10\%]) which happened in overall patients included meningitis, encephalitis, cerebral infarction, and brain abscess. Cardiovascular diseases (9\% [95\% CI 6\%-16\%]) which were reported in overall patients involved the myocarditis, endocarditis, valvular neoplasm, valvular perforation, pericardial effusion, and heart failure. Hemophagocytic syndrome (6\% [95\% CI $2 \%-23 \%])$ was only reported in adult patients. There were $13 \%$ of patients (95\% CI: 7\%-21\%) suffering from respiratory manifestations, including cough, pneumonia, bronchial pneumonia, pleural effusion, respiratory failure, and pulmonary embolism. Orchitis or epididymitis occurred in $9 \%$ of the male patients (95\% CI: 7\%-12\%).

3.4. Laboratory Tests. Table 4 shows the meta-analysis of the incidence of laboratory tests. There are 37 articles providing data of laboratory indicators of patients including 2999 cases. The mainly common reported abnormal laboratory tests were aleucocytosis (24.1\% [95\% CI 19.5\%-29.8\%]), anemia (23.9\% [95\% CI 18.5\%-30.9\%]), thrombocytopenia (15.8\% [95\% CI 
TABLE 4: Meta-analysis of the incidence of laboratory tests.

\begin{tabular}{lcr}
\hline Laboratory & The number of articles & Proportion [95\% CI] \\
\hline Thrombocytopenia & 32 & $0.158[0.1268 ; 0.1979]$ \\
Aleucocytosis & 37 & $0.241[0.1951 ; 0.2984]$ \\
Leukocytosis & 16 & $0.106[0.0819 ; 0.1365]$ \\
Anemia & 28 & $0.239[0.1847 ; 0.3094]$ \\
Pancytopenia & 6 & $0.132[0.093 ; 0.187]$ \\
\hline
\end{tabular}

12.7\%-19.8\%]), pancytopenia (13.2\% [95\% CI 9.3\%-18.7\%]), and leukocytosis (10.6\% [95\% CI 8.2\%-13.7\%]). The agglutination test was positive in $100 \%$ cases. Totally 30 articles including 4681 cases were tested by blood culture and Brucella melitensis species were isolated from $(48.3 \%$ [95\% CI $41.5 \%-56.3 \%]$ ) cases.

3.5. Misdiagnosis. There are 24 articles that provided information of misdiagnosis of patients including 2148 cases. 10 studies were from pastoral areas (3 from Xinjiang, 2 from Heilongjiang, 2 from Ningxia, 1 from Inner Mongolia, and 2 from Jilin) and 14 studies from nonpastoral areas (4 from Shandong, 3 from Beijing, 1 from Henan, 1 from Hebei, 1 from Hunan, 1 from Shanxi, 1 from Tianjin, 1 from Jiangxi, and 1 from Jiangsu). A total of 1287 (62.5\% [95\% CI $56.4 \%-69.2 \%]$ ) patients were misdiagnosed at the first visit (Figure 3 ). The misdiagnosed cases mainly occurred in nonpastoral provinces. Most patients were easily misdiagnosed as cold, rheumatic fever, rheumatoid arthritis, typhoid fever and paratyphoid fever, tuberculosis, malaria, septicemia, and lumbar disc herniation at the first visit in Department of Rheumatology, Hematology, Orthopedics, or Respiration.

\section{Discussion}

Brucellosis is one of the most widespread zoonoses worldwide $[82,83]$. The number of brucellosis patients is increasing year by year in China. Shi et al. [84] analyzed the incidence and spatial-temporal distribution of human brucellosis from 1955 to 2014 in China, and the report showed that human brucellosis had reemerged since the mid-1990s and the affected areas had expanded from northern pastureland provinces to southern coastal and southwestern areas since 2004. In China brucellosis has been increasingly causing huge economic loss, and it has been a population health problem in recent years.

In humans, brucellosis involved multiorgans with a complicated and various clinical presentations ranging from nonspecific to severe symptoms [85], which makes brucellosis easily misdiagnosed as other diseases. If a chronic phase is developed for a lack of timely diagnosis and treatment, the disease can lead to a high rate of disability. Since the clinical summary of the relevant cases in China is published in Chinese, these cases are not included in the study using metaanalysis of Brucella abroad [86]. We analyzed the literature of clinical manifestations of human brucellosis in China.

From our analyzed data, it shows that $57 \%$ selected studies from pastoral areas and 43\% from nonpastoral and coastal areas, consistent with previous epidemiological findings that the disease affected areas have expanded from northern pastureland provinces to southern coastal and southwestern areas over the past decades in China, but brucellosis is still mainly popular in pastoral areas [87]. In this study, there are $79.4 \%$ of patients who had close contact with sick animals and $11.5 \%$ of cases had consumption of uncooked meat or dairy products, indicating that contacting with infected animals and consuming unsterile animal products are the main transmission routes in China.

In the study, we found that the main clinical manifestations of human brucellosis are fever, fatigue, arthralgia, and muscle pain. The most common clinical syndromes of adult patients are fever, muscle pain, arthralgia, and sweating. Similar to our study, in a systematic review of the clinical manifestations of human brucellosis [86], the authors found that fever, arthralgia, myalgia, and back pain affected around half of the patients $(78 \%, 65 \%, 47 \%$, and $45 \%$, resp.). There is controversy about whether clinical manifestations in children are significantly different from manifestations in adults. It had been reported in the literature that there was no significant difference in clinical manifestations between children and adults [88], which was very different in different literature. Some scholars reported that enlarged lymph nodes, spleen and liver, skin rashes, pharyngitis [89], and hematological and respiratory complications were more frequently observed in children than in adults [90]. Children had higher rates of hepatitis, osteoarticular manifestations [91], and lower rates of meningitis, endocarditis, spondylitis, and the progression to chronicity [92]. In the study, we found clinical differences between children and adults. Children had higher rates of rash, respiratory and cardiac complications, and orchitis/epididymitis. We also noted that chills, headache, and weight loss are less frequently observed in children patients.

Multiorgan involvement of Brucella is probably underrecognised [93]. Bone, CNS, and epididymis are the most commonly included organs [6]. The results of the current study were similar to those in other reported articles [94]. In the current study, results show that hepatitis and osteoarthritis were the more frequent complications. Serious complications such as central nervous system dysfunction, cardiovascular diseases, respiratory manifestations, and hemophagocytic syndromes are also observed. Orchitis or epididymitis occurred in $9 \%$ of the male patients. Brucellosis complications remain a major medical problem and it must still be regarded as a serious health problem in China.

In the study, results show that there is a high rate of misdiagnosis that mainly occurred in nonpastoral areas. Because of these manifestations such as fever, back pain, 




FIGURE 3: Forest plot of the incidence of misdiagnosis.

cough, gastrointestinal symptoms, and blood abnormalities, brucellosis is often misdiagnosed. Most misdiagnosed patients were admitted in Department of Rheumatology, Hematology, Orthopedics, and Respiration at the first visit. Brucella bacteria culture is the "gold standard" for the diagnosis of brucellosis $[95,96]$. In the study, $87 \%$ of brucellosis patients have fever. However, we found that only 30 articles including 4681 cases were tested by blood culture and $48.3 \%$ of cases were positive of Brucella melitensis, indicating it may be the main reason of inappropriate diagnosis and inadequate therapy. Therefore, in order to reduce the rate of misdiagnosis effectively especially in the nonpastoral areas and provinces with high incidence of tuberculosis, it is necessary to broaden the ideas of clinical diagnosis with detailed history and carried out agglutination test and blood culture as early as possible for fever patients. One challenge in diagnosis of brucellosis is that the most common laboratory abnormalities are nonspecific. Most patients have normal blood cell counts on presentation. In the study, we found that the common abnormal laboratory tests were aleucocytosis $(24.1 \%)$, anemia (23.9\%), thrombocytopenia (15.8\%), pancytopenia (13.2\%), and leukocytosis (10.6\%). In case of pancytopenia, the diagnosis of secondary hemophagocytosis should be considered. This condition may be triggered by Brucella and other intracellular pathogens $[97,98]$.

Our study has some limitations. First, although the incidence of brucellosis is very high in our country, the quantity and quality of articles reported in some provinces are not high, which leads to partial data omission. We failed to obtain more precise analysis of different clinical stages of brucellosis because part of the included literature did not clearly describe the brucellosis clinical stage and age classification. Second, most of the reported literatures lack detailed data on patient treatment options and prognosis, which results in the failure of analyzing therapeutic effect and prognosis.

In summary, we found that brucellosis was mainly popular in pastoral areas, but the disease affected areas had expanded from northern pastureland provinces to southern coastal and southwestern areas in China. The infection is mostly associated with the contact with infected animals or through the consumption of raw animal products. Clinical symptoms include fever, fatigue, arthralgia, sweating, and muscle pain with complication such as osteoarthritis, hepatitis, central nervous system dysfunction, cardiovascular diseases, respiratory manifestations, orchitis or epididymitis, 
and hemophagocytic syndromes. Further research is needed to characterize the analysis for therapeutic effect and prognosis of brucellosis in China. Our study provides initial evidence for the accurate diagnosis.

\section{Conflicts of Interest}

The authors declared no potential conflicts of interest with respect to the research, authorship, and/or publication of this article.

\section{Acknowledgments}

This work was supported by Key Research and Development Projects of the Xinjiang Uygur Autonomous Region (no. 2016B03047-1).

\section{References}

[1] J. Ariza, M. Bosilkovski, A. Cascio et al., "Perspectives for the treatment of brucellosis in the 21st century: The Ioannina recommendations," PLoS Medicine, vol. 4, no. 12, pp. 1872-1878, 2007.

[2] G. Pappas, P. Papadimitriou, N. Akritidis, L. Christou, and E. V. Tsianos, "The new global map of human brucellosis," The Lancet Infectious Diseases, vol. 6, no. 2, pp. 91-99, 2006.

[3] M. F. De, G. A. Di, and A. Petrini, "Correlation between animal and human brucellosis in Italy during the period 1997-2002," Clinical Microbiology \& Infection, vol. 11, no. 8, Article ID 19972002, pp. 632-636, 2005.

[4] S. S. Mangalgi, A. G. Sajjan, S. T. Mohite, and S. Gajul, "Brucellosis in occupationally exposed groups," Journal of Clinical and Diagnostic Research, vol. 10, no. 4, pp. DC24-DC27, 2016.

[5] M. Moosazadeh, R. Nikaeen, G. Abedi, M. Kheradmand, and S. Safiri, "Epidemiological and clinical features of people with malta fever in iran: a systematic review and meta-analysis," Osong Public Health and Research Perspectives, vol. 7, no. 3, pp. 157-167, 2016.

[6] T. Buzgan, M. K. Karahocagil, H. Irmak et al., "Clinical manifestations and complications in 1028 cases of brucellosis: a retrospective evaluation and review of the literature," International Journal of Infectious Diseases, vol. 14, no. 6, pp. e469-e478, 2010.

[7] M. N. Seleem, S. M. Boyle, and N. Sriranganathan, "Brucellosis: A re-emerging zoonosis," Veterinary Microbiology, vol. 140, no. 3-4, pp. 392-398, 2010.

[8] S. Deqiu, X. Donglou, and Y. Jiming, "Epidemiology and control of brucellosis in China," Veterinary Microbiology, vol. 90, no. 1-4, pp. 165-182, 2002.

[9] J. Zhang, "Reasons and prevention and control measures for the high brucellosis incidence," Disease Surveillance, vol. 25, pp. 341$342,2010$.

[10] G. Gao, X. Jie, K. E. Yue-Hua et al., "Literature analysis of brucellosis epidemic situation and research progress in China from 2000 to 2010," Chinese Journal of Zoonoses, pp. 1178-1184, 2012.

[11] J. Zhang, F. Yin, T. Zhang et al., "Spatial analysis on human brucellosis incidence in mainland China: 2004-2010," BMJ Open, vol. 4, no. 4, Article ID e004470, 2014.
[12] S. Lai, H. Zhou, W. Xiong et al., "Changing epidemiology of human brucellosis, China, 1955-2014," Emerging Infectious Diseases, vol. 23, no. 2, pp. 184-194, 2017.

[13] Y.-J. Li, X.-L. Li, S. Liang, L.-Q. Fang, and W.-C. Cao, "Epidemiological features and risk factors associated with the spatial and temporal distribution of human brucellosis in China," BMC Infectious Diseases, vol. 13, no. 1, article no. 547, 2013.

[14] Y. Wu, X. Fei, R. Jiang et al., "Clinical manifestations and treatment of 44 patients with brucellosis," Infectious Disease Information, vol. 25, no. 1, pp. 22-25, 2012.

[15] L. Dai, J. Li, and X. Xu, "Retrospective analysis of clinical profiles of Brucellosis," Chinese Journal of General Practitioners, vol. 12, no. 7, pp. 562-563, 2013.

[16] Y. Ge, X. Liu, S. Zhang et al., "Clinical analysis for 66 cases of brucellosis in a general hospital," Chinese Journal of General Practitioners, vol. 10, no. 6, pp. 420-422, 2011.

[17] J. Tong, W. Zhao, J. Hu et al., "Clinical features of patients with brucellosis complicated by hemophagocytic syndrome," Infectious Disease Information, vol. 26, no. 1, pp. 32-38, 2013.

[18] X. Guo and Q. Xu, "Clinical analysis of 21 cases of Brucellosis in non-endemic area," Journal of China-Japm Friendship Hospital, vol. 27, no. 2, pp. 76-78, 2013.

[19] J. Wang, X. Hu, and L. Ma, "Epidemiological characteristics, Clinical manifestation and curative effect analysis of 61 cases of Brucellosis," Chinese Journal of Mycology, vol. 10, no. 5, pp. 288-290, 2015.

[20] Y. Gao, L. Suo, and W. Niu, "Analysis of clinical epidemiological characteristics of 182 brucellosis patients in Sunan County," Endemic Diseases Bulletin, vol. 17, no. 2, pp. 34-35, 2002.

[21] X. Zhang, J. Wang, P. Li et al., "Epidemiological and clinical data analysis for 21 cases of brucellosis patients," China Modern Doctor, vol. 50, no. 5, pp. 4-5, 2012.

[22] Y. Li, J. Zhang, Q. Xie et al., "Epidemiological and clinical characteristics of 905 cases with brucellosis, Pingdingshan, 2014," Modern Preventive Medicine, vol. 43, no. 1, pp. 21-23, 2016.

[23] J. Zhou, "Incidence and clinical characteristics of 241 cases of brucellosis in Luoyang," Henan Journal of preventive Medicine, vol. 20, no. 4, pp. 276-277, 2009.

[24] S.-C. Li, J.-W. Yu, J. Gao, and L.-J. Sun, "Retrospective analysis of epidemiological and clinical features of patients with acute brucellosis in Harbin and nearby counties over the past twenty years," Chinese Journal of Endemiology, vol. 27, no. 6, pp. 691693, 2008.

[25] L. Liu and M. Zhang, "Observation of clinical manifestations and treatment of 44 cases of brucellosis," China Rural Health, vol. 11, pp. 42-43, 2016.

[26] C. Liu, Y. Zhang, and W. Liu, "Clinical analysis of 314 cases of brucellosis," International Journal of Epidemiology and Infectious Disease, vol. 42, no. 5, pp. 347-348, 2015.

[27] D. Meng, W. Liu, Z. Meng et al., "Epidemiological characteristics and clinical analysis of human brucellosis in Heilongjiang province from 2010 to 2013," Chinese Jouranl of Endemiology, vol. 34, no. 7, p. 530, 2015 (Chinese).

[28] A. Gong, H. Yan, Z. Xu et al., "Analysis of the clinical character of brucellosis," International Journal of Epidemiology and Infectious Disease, vol. 37, no. 1, pp. 16-18, 2010.

[29] J. Liu, C. Fu, and X. Chen, "Clinical analysis of 229 cases of brucellosis," Journal of Zhejiang University (Medical Sciences), vol. 41, no. 6, pp. 677-680, 2012.

[30] L. Sun, Z. Zhang, S. Cong et al., "Analysis of population distribution and clinical syndrome of 270 cases of patients with 
brucellosis," Modern Preventive Medicine, vol. 37, no. 15, pp. 2998-2999, 2010.

[31] K. Wang, Z. Zhang, X. Liu et al., "Analysis of the Epidemiologic \& Clinical Characteristics of 88 Cases of Brucellosis," Journal of Microbiology, vol. 34, no. 4, pp. 97-100, 2014.

[32] Y. Xie, E. Jia, Y. Kang et al., "Analysis of 166 cases of brucellosis epidemiological and clinical features," Journal of Diseases Monitor \& Control, vol. 10, no. 11, pp. 870-871, 2016.

[33] Q. Sheng and M. Ma, "Clinical analysis of 829 cases of brucellosis," China Morden Medicine, vol. 16, no. 25, p. 147, 2009.

[34] M. Sun, Z. Ma, and Z. Bao, "Brucellosis epidemiological and clinical features: 126 cases analysis," Chinese Journal of Difficult and Complicated Cases, vol. 13, no. 3, pp. 271-274, 2014.

[35] W. Yang and F. Yang, "To investigate the epidemiology of brucellosis, clinical and laboratory features," Journal of Clinical Medical Literature, vol. 2, no. 3, pp. 465-468, 2015.

[36] J. Duan, "A retrospective study of 57 cases of brucellosis," Medical Information, vol. 28, no. 43, pp. 379-380, 2015 (Chinese).

[37] A. Zhang and Y. Wang, "Analysis of clinical characteristics of brucellosis in Ningxia," China Practical Medicine, vol. 8, no. 29, pp. 46-47, 2013.

[38] Z. Wang, "Analysis of clinical characteristics and epidemiological of 62 patients with brucellosis," Practical Preventive Medicine, vol. 12, no. 2, p. 430, 2005 (Chinese).

[39] Q. Wang and Y. Xiong, "Analysis of clinical characteristics of brucellosis in 235 cases," Journal of China Traditional Chinese Medicine Information, vol. 3, no. 23, p. 197, 2011.

[40] Y. Lian, X. Wang, and M. Wang, "Clinical characteristics of brucellosis patients at different ages," Chinese Journal of Infectious Diseases, vol. 33, no. 3, pp. 164-165, 2015.

[41] D. Gao, "The monitoring of Brucella and clinical analysis of human brucellosis in the southwest of Shandong Province," Laboratory Medicine, vol. 31, no. 7, pp. 571-575, 2016.

[42] X. Wang, Z. Zeng, Q. Qu et al., "Clinical features and diagnosis and treatment process of 96 cases with brucellosis in Shandong Province," Chinese Journal of Experimental and Clinical Infectious Disease, vol. 8, no. 4, pp. 548-551, 2014.

[43] Q. Wang, "Clinical analysis of 86 cases of brucellosis," Proceeding of Clinical Medicine, vol. 19, no. 12, pp. 926-927, 2010.

[44] S. Feng and C. Deng, "Survey of clinical epidemiological of 105 brucellosis cases in Shanxi," Chinese Journal of Infectious Diseases, vol. 34, no. 1, pp. 43-45, 2016.

[45] S. An, A. Han, and Y. Cui, "Analysis of clinical epidemiological characteristics of 622 patients with brucellosis," Chinese Journal of Epidemiology, vol. 22, no. 5, p. 329, 2001.

[46] G. Zhang, H. Su, L. Yang et al., "Clinical analysis of 43 cases with brucellosis," Chinese Journal of Primary Medicine and Pharmacy, vol. 23, no. 17, pp. 2024-2026, 2016.

[47] S. Guo, X. Luo, and R. Gou, "Clinical characteristics of 124 cases of patients with brucellosis," China Tropical Medicine, vol. 16, no. 1, pp. 93-94, 2016.

[48] K. Pan, M. Wubuli, B. Ababaikeli et al., "Analysis of 153 patients with brucellosis in Xinjiang Uyghur Autonomous Region," Infectious Disease Information, vol. 26, no. 1, pp. 47-49, 2013.

[49] Y. Zhang and L. Liu, "Epidemiological and clinical features of 57 cases with brucellosis," Chinese Journal of Experimental and Clinical Infectious Disease (Electronic Version), vol. 7, no. 4, pp. 563-565, 2013.

[50] X. Yang, J. Wu, and D. Sun, "linical analysis of discharged cases of brucellosis in our hospital from 2013 to 2014," Xinjiang Medical Journal, vol. 45, no. 5, pp. 631-635, 2015.
[51] Y. Zhang, "Clinical Analysis of 191 Cases of Undulant Disease in Beitun Hospital," Xinjiang Medical Journal, vol. 46, no. 3, pp. 326-340, 2016.

[52] L. Ju, X. Geng, and Z. Huang, "Clinical analysis of 156 cases of brucellosis," Clinical Misdiagnosis \& Mistherapy, vol. 24, no. 5, pp. 14-16, 2011.

[53] H. Gao, X. Zhang, L. Zu et al., "Analysis of clinical syndrome and integrated traditional Chinese and Western medicine treatment of brucellosis," Global Chinese Medicine, vol. 5, no. 6, pp. 461462, 2012.

[54] Y. Wang, X. Sun, Y. Xiang et al., "Clinical analysis of 117 patients with brucellosis in Xinjiang Uyghur Autonomous Region," Infectious Disease Information, vol. 28, no. 1, pp. 38-42, 2015.

[55] W. Wang, X. Feng, Y. Tian et al., "Clinical treatment and analysis of Children's brucellosis," Hebei Medical Journal, vol. 36, no. 23, pp. 3635-3636, 2014.

[56] Q. Zeng, Y. Li, Y. Meng et al., "Clinical retrospective analysis on children with brucellosis," Maternal and Child Health Care of China, vol. 29, pp. 1710-1713, 2014.

[57] Y. Wang, Y. Gao, and X. Sun, "Clinical characteristics of brucellosis in pediatric patients in Xinjiang Uyghur Autonomous Region," Infectious Disease Information, vol. 29, no. 1, pp. 39-46, 2016.

[58] R. Fan, S. Yue, Y. Zhang et al., "The clinical features and treatment of Brucellosis disease in 24 children," Journal of Clinical Pediatrics, vol. 34, no. 10, pp. 744-746, 2016.

[59] Z. Zhang, L. Zhang, and Y. Liu, "Clinical characteristics and treatment of acute brucellosis in 25 children," Chinese Journal of Infection Control, vol. 21, no. 2, pp. 112-113, 2006.

[60] Z. Yu, D. Cheng, Y. Chen et al., "Clinical analysis of 38 cases of acute brucellosis in children," Chinese Journal of Endemiology, vol. 31, no. 2, p. 230, 2012.

[61] M. Lu and R. Liu, "Analysis of brucellosis among children in Wulanchabu of Inner Mongolia from 2011 to 2013," Chinese Journal of Endemiology, vol. 34, no. 5, p. 385, 2015.

[62] C. Liu, Y. Zhang, and J. He, "Clinical features of children with brucellosis," International Journal of Epidemiology and Infectious Disease, vol. 43, no. 4, pp. 236-239, 2016.

[63] H. Bai and Z. Duan, "Analysis of clinical characteristics of 48 children with brucellosis," Ningxia Medical Journal, vol. 37, no. 12, pp. 1190-1191, 2015.

[64] F. He, "Clinical analysis of 19 cases of brucellosis in children," Chinese Pediatric Emergency Medicine, vol. 22, no. 9, pp. 640642, 2015.

[65] G. Zheng, C. Zeng, and Y. Jiang, "Clinical analysis of 12 cases of brucellosis in non pastoral areas," Chinese Journal of Hygiene Rescue, vol. 2, no. 4, pp. 230-232, 2016.

[66] C. Tong, L. Wang, Y. Cai et al., "Analysis of 25 cases of brucellosis in acute stage," Chinese Journal of Misdiagnostics, vol. 8, no. 15, pp. 3732-3733, 2008.

[67] Y. Chen and Y. Dong, "Exploration on experience of clinical manifestations and clinical diagnosis and treatment of brucellosis," Journal of Clinical Medical, vol. 3, no. 12, pp. 2277-2280, 2016.

[68] Q. Huang, "Epidemiological investigation and clinical analysis of 17 cases of brucellosis," China's Rural Health, vol. 16, p. 27, 2016.

[69] L. Ji, Y. Zhao, and J. Xu, "Analysis of 30 cases of brucellosis," Chinese Journal of Misdiagnosis, vol. 6, no. 17, p. 3419, 2006.

[70] M. Wang and L. Wang, "Clinical analysis of 26 cases of brucellosis," Chinese Journal of Infection Control, vol. 22, no. 4, pp. 286-287, 2007. 
[71] L. Zhang, L. Deng, X. Jiang et al., "Clinical and laboratory analysis of brucellosis in 39 patients in Northern Jiangsu Province," International Journal of Laboratory Medicine, vol. 37, no. 15, pp. 2052-2054, 2016.

[72] L. Guo, B. Tong, Z. Xiao et al., "Diagnosis and treatment of brucellosis in Jiangxi Province," Jiangxi Medical Journal, vol. 51, no. 8, pp. 796-798, 2016.

[73] X. Zhang, "Clinical analysis of 27 cases of brucellosis," Chinese Journal of Postgraduates of Medicine, vol. 34, no. 4, pp. 43-44, 2011.

[74] Y. Yan, B. Duan, P. Wu et al., "Analysis of 31 cases of brucellosis infection," Journal of Ningxia Medical University, vol. 38, no. 5, pp. 556-558, 2016.

[75] Z. Li, X. Cao, L. Jiang et al., "Clinical characteristics of patients with brucellosis," Chinese Journal of Nosocomiology, vol. 25, no. 3, pp. 626-628, 2015.

[76] J. Wu, W. Wang, and L. Yang, "Clinical analysis of 28 cases of brucellosis," Journal of Changzhi Medical College, vol. 21, no. 6, pp. 433-434, 2007.

[77] H. Zhang and Y. Li, "Clinical and epidemiological analysis of 35 cases of brucellosis in non endemic areas," Shaanxi Medical Journal, vol. 44, no. 7, pp. 847-848, 2015.

[78] J. Wang, "Analysis of 17 cases of brucellosis," Tianjin Medical Journal, vol. 42, no. 3, pp. 280-282, 2014.

[79] Q. Zhou and W. Yang, "Clinical analysis of 18 cases of brucellosis with initial symptom of fever in non-pasture area," Chinese Journal of Infectious Diseases, vol. 32, no. 4, pp. 229-232, 2014.

[80] W. Xu, Y. Yang, X. Chen et al., "Clinical epidemiological characteristics of brucellosis patients in Zhejiang," Chinese Journal of Preventive Medicine, vol. 8, no. 1, pp. 68-69, 2007.

[81] G. Chen, L. Xu, Y. Qi et al., "Clinical analysis of 74 cases of the first-treated patients with brucellosis," Jilin Medical Journal, vol. 37, no. 4, pp. 793-795, 2016.

[82] A. S. Dean, L. Crump, H. Greter, E. Schelling, and J. Zinsstag, "Global burden of human brucellosis: a systematic review of disease frequency," PLOS Neglected Tropical Diseases, vol. 6, no. 10, Article ID e1865, 2012.

[83] M. P. Rubach, J. E. B. Halliday, S. Cleaveland, and J. A. Crump, "Brucellosis in low-income and middle-income countries," Current Opinion in Infectious Diseases, vol. 26, no. 5, pp. 404412, 2013.

[84] Y. Shi, S. Lai, Q. Chen et al., "Analysis on the epidemiological features of human brucellosis in northern and southern areas of China, 2015-2016," Zhonghua Liu Xing Bing Xue Za Zhi, vol. 38, no. 4, pp. 435-440, 2017.

[85] A. Ulu-Kılıç, G. Metan, and E. Alp, "Clinical presentations and diagnosis of brucellosis," Recent Patents on Anti-Infective Drug Discovery, vol. 8, no. 1, pp. 34-41, 2013.

[86] A. S. Dean, L. Crump, H. Greter, J. Hattendorf, E. Schelling, and J. Zinsstag, "Clinical manifestations of human brucellosis: a systematic review and meta-analysis," PLOS Neglected Tropical Diseases, vol. 6, no. 12, Article ID e1929, 2012.

[87] P. Jia and A. Joyner, "Human brucellosis occurrences in inner mongolia, China: A spatio-temporal distribution and ecological niche modeling approach," BMC Infectious Diseases, vol. 15, no. 1, p. 36, 2015.

[88] S. Ahmetagic, N. Tihic, A. Ahmetagic et al., "Human brucellosis in Tuzla Canton.", Medical Archives, vol. 66, no. 5, pp. 309-314, 2012.

[89] A. M. Yinnon, G. A. Morali, A. Goren et al., "Effect of age and duration of disease on the clinical manifestations of brucellosis:
A study of 73 consecutive patients in Israel," Israel Journal of Medical Sciences, vol. 29, no. 1, pp. 11-16, 1993.

[90] A. Gür, M. F. Geyik, B. Dikici et al., "Complications of brucellosis in different age groups: a study of 283 cases in southeastern Anatolia of Turkey," Yonsei Medical Journal, vol. 44, no. 1, pp. 33-44, 2003.

[91] O. Megged, B. Chazan, A. Ganem et al., "Brucellosis outbreak in children and adults in two areas in Israel," American Journal of Tropical Medicine \& Hygiene, vol. 95, no. 1, pp. 31-34, 2016.

[92] M. Bosilkovski, V. Kirova-Urosevic, Z. Cekovska et al., "Osteoarticular involvement in childhood brucellosis: Experience with 133 cases in an endemic region," The Pediatric Infectious Disease Journal, vol. 32, no. 8, pp. 815-819, 2013.

[93] J. A. Herrick, R. J. Lederman, B. Sullivan, J. H. Powers, and T. N. Palmore, "Brucella arteritis: Clinical manifestations, treatment, and prognosis," The Lancet Infectious Diseases, vol. 14, no. 6, pp. 520-526, 2014.

[94] P. De Figueiredo, T. A. Ficht, A. Rice-Ficht, C. A. Rossetti, and L. G. Adams, "Pathogenesis and immunobiology of brucellosis review of Brucella- host interactions," The American Journal of Pathology, vol. 185, no. 6, pp. 1505-1517, 2015.

[95] A. Barua, A. Kumar, D. Thavaselvam et al., "Isolation \& characterization of brucella melitensis isolated from patients suspected for human brucellosis in India," The Indian Journal of Medical Research, vol. 143, pp. 652-658, 2016.

[96] S. Mangalgi and A. Sajjan, "Comparison of three blood culture techniques in the diagnosis of human Brucellosis," Journal of Laboratory Physicians, vol. 6, no. 1, pp. 14-17, 2014.

[97] A. Cascio, L. M. Pernice, G. Barberi et al., "Secondary hemophagocytic lymphohistiocytosis in zoonoses. A systematic review," European Review for Medical and Pharmacological Sciences, vol. 16, no. 10, pp. 1324-1337, 2012.

[98] A. Cascio, S. Giordano, P. Dones, S. Venezia, C. Iaria, and O. Ziino, "Haemophagocytic syndrome and rickettsial diseases," Journal of Medical Microbiology, vol. 60, no. 4, pp. 537-542, 2011. 


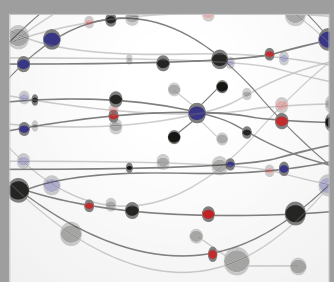

The Scientific World Journal




Gastroenterology Research and Practice

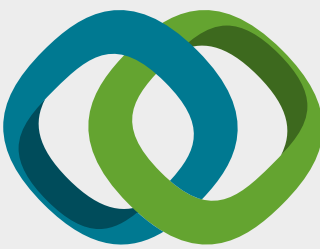

\section{Hindawi}

Submit your manuscripts at

www.hindawi.com
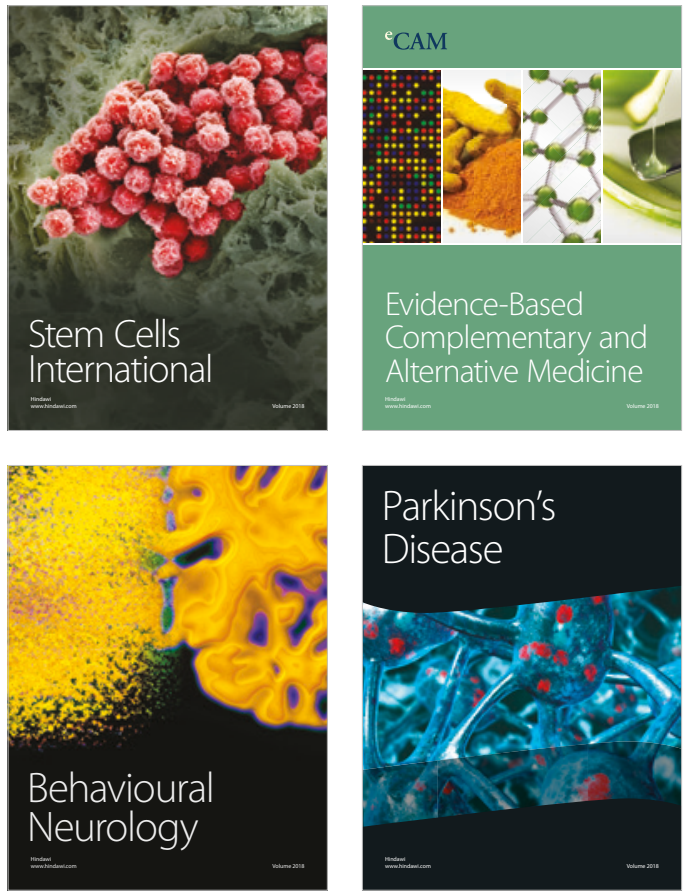



ournal of

Diabetes Research

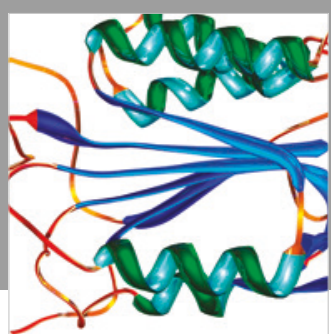

Disease Markers
